$$
\mathrm{CH}_{3} \cdot \mathrm{CO} \cdot \mathrm{C}_{2} \mathrm{H}_{5}^{\nearrow} \underset{\mathrm{C}_{2} \mathrm{H}_{5} \cdot \mathrm{CO} \cdot+\mathrm{CH}_{3} .}{\mathrm{CH}_{3} \cdot \mathrm{CO} \cdot+\mathrm{C}_{2} \mathrm{H}_{5} \cdot}
$$

A preliminary investigation at a longer wave-length (about 3100 A.) indicates that this ratio then becomes considerably greater.

The method is obviously of general applicability, and is being extended to the investigation of other free radical reactions; a fuller report will be published elsewhere. Our thanks are due to Prof. F. A. Paneth for his continued interest, and to the Isotope Division, Atomic Energy Research Establishment, Harwell, for the supply of radioactive material.

R. W. DURHAM

G. R. MARTIN

H. C. SUtToN

Londonderry Laboratory for Radiochemistry, Durham. July 26.

${ }^{1}$ I eigh ton, P. A., J. Amer. Chem. Soc., 58, 448 (1936). See also Burton, M., Ricei, J. E., and Davis, T. W., ibid., 62, 265 (1940) and Harris, G. M., and Tickner, A. W., J.'Chem. Phys., 15, 686 (1947).

2 v. Hartel, H., and Polanyi, M., Z. physik. Chem., B, 11, 97 (1930). ${ }^{3}$ Gorin, E., Acta Physicochimica U.R.S.S., 8, 513 (1938).

\section{Chromic Acid Oxidation of Ethyl Alcohol}

IN connexion with a problem of biosynthesis, we had occasion to prepare a sample of ethanol in which some of the $\alpha$-hydrogen atoms were replaced by deuterium. This was done by heating propionic acid with deuterium sulphate, recovering the labelled propionic acid and treating its silver salt with bromine in carbon tetrachloride; the resulting ethyl bromide was hydrolysed by heating with water or with aqueous silver sulphate. Absence of deuterium from the methyl group of this alcohol was proved by oxidation to acetic acid with aqueous potassium permanganate. The silver salt of this acetic acid contained very little deuterium (0.03-0.06 per cent excess), and this was not due to exchange with the aqueous medium during oxidation, for a parallel experiment on ordinary ethanol in a heavy water medium also afforded deuterium-free silver acetate. Analysis of the labelled ethanol as ethyl 3:5-dinitrobenzoate indicated a deuterium excess of 40 'atom per cent' on the two $\alpha$-hydrogen atoms. The sample was thus a mixture of $\mathrm{CH}_{3} \mathrm{CH}_{2} \mathrm{OH}, \mathrm{CH}_{3} \mathrm{CHDOH}$ and $\mathrm{CH}_{3} \mathrm{CD}_{2} \mathrm{OH}$.

Some of this ethanol was oxidized with aqueous chromic acid, and acetaldehyde was isolated as the $p$-nitrophenylhydrazone. Analysis of this derivative indicated, surprisingly, that the acetaldehyde contained 60 per cent of $\mathrm{CH}_{3} \mathrm{CDO}$. The unlikely possibility that intramolecular shift of deuterium to the methyl group had occurred during oxidation was eliminated by oxidizing part of the acetaldehyde to acetic acid : the derived silver acetate was free from deuterium. In three oxidation experiments on the ethanol (two with chromium trioxide and one with sodium dichromate-sulphuric acid) the deuterium content of the acetaldehyde was much the same (58-62 per cent $\mathrm{CH}_{3} \mathrm{CDO}$ ).

Ethanol recovered from an oxidation showed little change in isotopic content (38 atom per cent excess deuterium in the $\alpha$-positions, compared with the original 40). However, when acetaldehyde containing $27 \cdot 1$ per cent $\mathrm{CH}_{3} \mathrm{CDO}$ was treated with warm chromic acid, the acetaldehyde recovered contained $32 \cdot 6$ per cent $\mathrm{CH}_{3} \mathrm{CDO}$, although the proportion of aldehyde oxidized seemed relatively small.

It is clear that $\mathrm{CH}_{3} \mathrm{CHO}$ is destroyed considerably faster by chromic acid than is $\mathrm{CH}_{3} \mathrm{CDO}$. This effect may be largely responsible for the remarkable 'deuterium enrichment' in the oxidation of ethanol to acetaldehyde; but it is still possible that preferential elimination of hydrogen may take place at some stage of acetaldehyde formation.

The experiments will be described elsewhere in detail. We are indebted to Miss Marie-Louise Beeckmans for help with deuterium analyses.

\section{J. W. CORNFORTH}

G. Popják

National Institute for Medical Research, London, N.W.3. July 28.

\section{Synthesis of a Physiologically Active Com- pound of the Pellitorine Structure}

PeLirtorine, isolated from the roots of pellitory, Anacyclus pyrethrum, has aroused renewed interest since it has been shown to possess insecticidal activity. It has been stated by Jacobson' ${ }^{1}$ to possess a 'knockdown value' against house-flies equal to, and a toxicity somewhat more than half, that of the pyrethrins tested at the same concentration. Pellitorine (m.p. $72^{\circ}$ ) was shown by Gulland and Hopton ${ }^{2}$ to be the isobutylamide of a $n$-nonadiene-1-carboxylic acid, and Jacobson ${ }^{1}$ has since located the two double bonds in the $1: 2$ and $5: 6$ positions. Very recently, Raphael and Sondheimer ${ }^{3}$ have reported the synthesis of a liquid geometrical isomer of pellitorine, the cis-cis; while Jacobson" has prepared another geometrical isomer (m.p. $54-55^{\circ}$ ) of unspecified configuration. Neither of these isomers possesses the pungency and the sialogogue characteristic of natural pellitorine and that of Jacobson is non-toxic to house-flies. These reports prompt us to describe briefly our own progress in this field.

We had independently developed a similar syn. thesis to that of Jacobson, and from our study of the ring fission of 3-chloro-2-alkyltetrahydropyrans ${ }^{5}$ consider that Jacobson's product is the trans-trans isomer. Furthermore, we have now prepared the isobutylamide of n-nona-trans-1-cis-5-diene-1-carboxylic acid by the following route (the yields for each stage are indicated) :

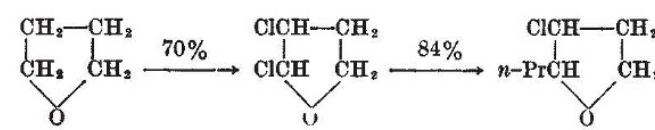

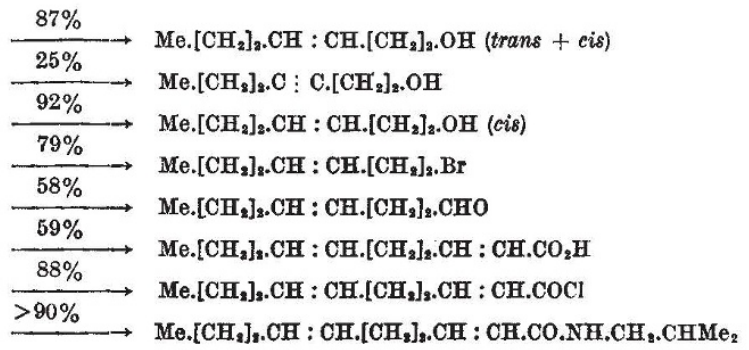

Chlorination of tetrahydrofuran gave 2 : 3-dichlorotetrahydrofuran, which was treated with $n$-propylmagnesium bromide to give a cis-trans mixture of 3-chloro-2-n-propyltetrahydrofuran, and this on ring 